\title{
Frequency-Domain Orthogonal Multiuser Communications over Frequency-Selective Slowly Fading Channels
}

\author{
Cha'o-Ming Chang \\ lepidus@santos.ee.ntu.edu.tw \\ Kwang-Cheng Chen \\ chenkc@cc.ee.ntu.edu.tw \\ Institute of Communications Engineering, National Taiwan University, Taiwan, R.O.C.
}

\begin{abstract}
Inheriting the properties of the frequency-domain approach [1] to avoid MAI in the asynchronous multiuser communications and sharing the rationale behind OFDM systems to mitigate ISI, we propose the asynchronous multiuser communications systems over frequency-selective slowly fading channels such that the optimal demodulation with similar complexity to pointto-point communications is possible. In addition, by spreading the symbol energies to the whole transmission frequency band, we introduce another asynchronous multiuser communications systems to utilize the frequency diversity gain. These proposed systems not only mitigate MAI and ISI efficiently with simple complexity but also are flexible for variable rates transmission to suggest their applications to practical multimedia services.
\end{abstract}

\section{INTRODUCTION}

One of the most challenging issues in the wireless communications systems is the mitigation of interference to improve the communications performance. In the direct sequence code division multiple access (DS-CDMA) multiuser communications systems, all users transmit information spread by their assigned different signature waveforms in the same frequency band simultaneously, and users are interfered by the other users known as the multiple access interference (MAI). Starting from Verdu's pioneer work [2], a great amount of researchers have been working on the multiuser detection to minimize the impact of MAI to DS-CDMA multiuser communications. By taking the feasible implementation into consideration, however, these approaches are of suboptimal performance and are prevailed by conventional detection employing timedomain orthogonal signature sequences (such as the WalshHadamard sequences).

It is required to maintain synchronous channels perfectly such that the conventional and simple demodulation approach is possible by employing the time-domain orthogonal signature sequences. The communications channels are said to be synchronous when the received timings from all users are the same and the demodulation performance degrades severely whenever the received timings are not perfectly aligned [3]. In the reverse link (also known as the up-link) transmission wherein the stations simultaneously transmit their signals to the base station, the maintenance of synchronous channel is not an easy task.

To solve this problem, we proposed the frequency-domain approach to DS-CDMA multiuser communications systems over additive white Gaussian noise (AWGN) channels [1].
By employing the novel but equivalent spreading approach and by utilizing the frequency-domain orthogonal signature sequences, the signature sequences are orthogonal even in the asynchronous channels such that the simple decoding approach is realized with optimal performance. The rationale behind this approach is to maintain signature sequences orthogonal over frequency domain instead of time domain, because the timing shift corresponds to the scaler multiplication over frequency domain. And therefore the signature sequences remain orthogonal in the asynchronous channels.

By extending this frequency-domain approach to frequencyselective slowly fading channels [4], this frequency-domain approach is interfered by inter-symbol interference (ISI) introduced by frequency-selective channels. The frequencyselective slowly fading channels can be modelled as a tapped delay line [5] and therefore the received signal is interfered by its delayed copies, known as ISI. On the other hand, unlike the

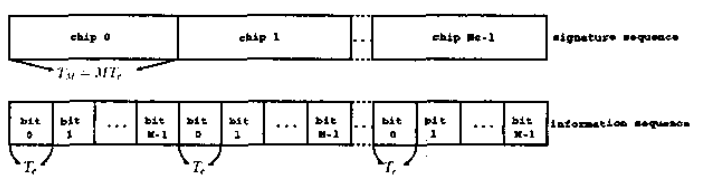

3

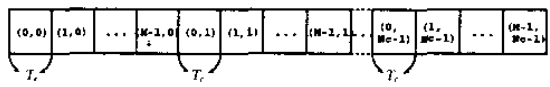

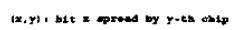

Fig. 1. Novel signal spreading in frequency-domain approaches.

conventional direct sequence spreading approach, each symbol in the frequency-domain orthogonal spreading is first spread by the signature sequence and then uniformly interleaved into the whole frame as shown in Figure 1. This feature makes the frequency-domain approach more vulnerable to ISI as the effective symbol length is reduced from $T$ to $T_{c}$, where $T \cong N_{c} T_{c}$. Fortunately, this frequency-domain approach is still not interfered by MAI.

In this paper, sharing the similar rationale behind the orthogonal frequency division multiplexing (OFDM) technologies to mitigate ISI, we shall introduce a family of frequency-domain orthogonal multiuser communications systems to combat the ISI with simple complexity. 
II. Frequency-Domain ORThogonal Multiuser COMMUNICATIONS WITH INVERSE TRANSFORMATION (FDOMC-IT) OVER FREQUENCY-SELECTIVE SLOWLY FADING CHANNELS

\section{A. Signal Models and Assumptions}

We consider the asynchronous multiuser communications systems with $K$ users over frequency-selective slowly fading channels such that the channel impulse responses of all users are time-invariant within a couple of frames. In addition, assuming that the null-to-null bandwidth of the transmitted signal is $W$, the frequency-selective slowly fading channel for user $k$ can be modelled as the tapped delay line having $L_{k}$ taps with tap spacing $1 / W$ [5].

Suppose that each user transmits $M_{\text {info }}$ information symbols in a frame. For example, $\left\{\tilde{B}_{k}^{\left(m^{\prime}\right)}(m) \mid m=0,1, \cdots\right.$, $\left.M_{\text {info }}-1\right\}$ for $k=0,1, \cdots, K-1$ are the $M_{\text {info }}$ information symbols of user $k$ in the $m^{\prime}$ th frame, where $m^{\prime} \in \mathcal{Z}$ and $\mathcal{Z}$ denotes the field of integers. We assume that the $k$ th user employs the binary phase shift keying (BPSK) modulation (i.e. $\left.\tilde{B}_{k}^{\left(m^{\prime}\right)}(m) \in \pm 1\right)$ though the results in the sequel are equally applicable to other linear modulated signals. It is remarkable to note that different users could use different modulation schemes without affecting the receiving performance of other users such that the data rates could be variable for users.

Before transmission, we first take $M_{\text {info-point inverse dis- }}$ crete Fourier transform (IDFT) on the information symbols and have $\tilde{b}_{k}^{\left(m^{\prime}\right)}(m) \equiv \frac{1}{M_{\text {info }}} \sum_{n=0}^{M_{\text {info }}-1} \tilde{B}_{k}^{\left(m^{\prime}\right)}(n) e^{j \frac{2 \pi}{M i_{\text {info }}} n m}$, for $m=0,1, \cdots, M_{\text {info }}-1$ and $k=0,1, \cdots, K-1$. In addition, for each user, we insert $M_{g}$ guard symbols before spreading, where these $M_{g}$ symbols are the cyclic prefix of the information symbols after performing IDFT and $M_{g}$ is a non-negative integer such that $M_{g} T_{c} \geq \max _{k=0,1, \cdots, K-1} \frac{L_{k}}{W}$, wherein $T_{c}$ is the chip time of the signature sequences. In order to simplify the illustrations, we assume $M_{\text {info }} \geq M_{g}$ for the rest of this paper. For the special case that $M_{g}>M_{\text {info }}$, we only need to pretend that the number of information symbols is $M_{g}$ by padding " 0 "s after $M_{\text {info }}$ information symbols. By employing the frequency-domain orthogonal spreading in [1], the $m$ th transmitted frame from user $k$ with $M \equiv M_{\text {info }}+M_{g}$ symbols is $\tilde{s}_{k}^{\left(m^{\prime}\right)}(t)=\sum_{n=0}^{N_{c}-1} p_{k}(n) \sum_{m=0}^{M-1} \ddot{b}_{k}^{\left(m^{\prime}\right)}(m) \Pi(t-$ $\left.m T_{c}-n T_{M}\right)$, where $p_{k}(n) \equiv e^{j \frac{2 \pi}{N_{c}} n k}$ for $n=0,1, \cdots, N_{c}-1$ is the signature sequence of user $k ; N_{c}$ is the number of chips within a signature sequence such that $N_{c} \geq K ; T_{M} \equiv M T_{c}$; $\Pi(t)$ is the indicator function with support $\left[0, T_{c}\right)$; and

$\ddot{b}_{k}^{\left(m^{\prime}\right)}(m) \equiv \begin{cases}\tilde{b}_{k}^{\left(m^{\prime}\right)}\left(m+M_{\text {info }}-M_{g}\right) & \text { if } 0 \leq m<M_{g} \\ \tilde{b}_{k}^{\left(m^{\prime}\right)}\left(m-M_{g}\right) & \text { if } M_{g} \leq m<M\end{cases}$

for $m=0,1, \cdots, M-1$ is the transmitted sequence of user $k$ after guard symbols insertion.

Let $T_{d i f f}$ be the maximal difference between the received timings of users. That is, $T_{d i f f} \equiv \max _{k, k^{\prime}=0,1, \cdots, K-1} \mid \tau_{k}-$ $\tau_{k^{\prime}} \mid$, where $\tau_{k}$ is the received timing of user $k$. And define
$T_{G} \equiv T_{\text {diff }}+\max _{k=0,1, \cdots, K-1} \frac{L_{k}}{W}$, which is the maximal possible distance in time between users considering the frequency-selective channels. In order to avoid the inter-frame interference, before transmitting $\tilde{s}_{k}^{\left(m^{\prime}\right)}(t)$, we further insert another guard time before each frame, which is the cyclic prefix of $\tilde{s}_{k}^{\left(m^{\prime}\right)}(t)$ with duration $T_{G}$. To ease the development, we temporarily assume that $T_{G}$ is smaller than $M T$. Therefore, the $m$ th frame of user $k$ with cyclic prefix between frames can be written as

$\ddot{s}_{k}^{\left(m^{\prime}\right)}(t)=\left\{\begin{array}{lc}\tilde{s}_{k}^{\left(m^{\prime}\right)}\left(t+M T-T_{G}\right) & 0 \leq t<T_{G} \\ \tilde{s}_{k}^{\left(m^{\prime}\right)}\left(t-T_{G}\right) & T_{G} \leq t<M T+T_{G} \\ 0 & \text { otherwise. }\end{array}\right.$

The frame structure and transmitter of this approach are plotted in Figure 2 and Figure 3(a).

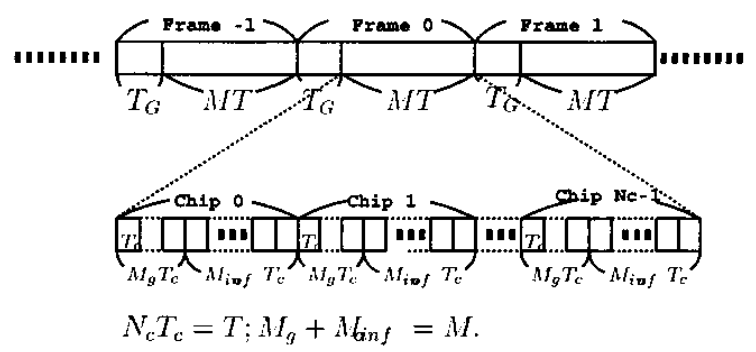

Fig. 2. Frame structure of frequency-domain orthogonal multiuser communications systems.

As the frames can be independently demodulated with guard intervals between frames, we demodulate frame 0 in the sequel as an example. The received low-pass equivalent signal $r_{L P}(t)$ with $K$ users in the frequency-selective slowly fading channels is $r_{L P}(t)=\sum_{k=0}^{K-1} \sum_{l=0}^{L_{k}-1} \rho_{k, l} \ddot{s}_{k}^{(0)}\left(t-\tau_{k}-\right.$ $\left.\frac{l}{W}\right)+z_{L P}(t)$, for $t \in\left[T_{G}+\min _{k=0,1, \cdots, K-1} \tau_{k}, M T+\right.$ $T_{G}+\min _{k=0,1, \cdots, K-1} \tau_{k}$ ), where $\rho_{k, l}$ is the complex-valued random variable whose magnitude (resp. phase) stands for the received amplitude (resp. phase) of user $k$ at the $l$ th tap; and $z_{L P}(t)$ is the low-pass equivalent additive white Gaussian noise whose two-sided power spectral density is $N_{0} / 2$.

Assume that the multiuser timing recovery (such as the one in [6]) and channel estimation schemes are available to the receiver such that $\tau_{k}$ and $\rho_{k, l}$ are known for all $k$ and $l$. Without loss of generality, by letting $0 \equiv \tau_{0} \equiv \min _{k=0,1, \cdots, K-1} \tau_{k} \leq$ $\max _{k=0,1, \cdots, K-1} \tau_{k} \equiv \tau_{K-1}$ and defining $t^{\prime} \equiv t-T_{G}$, we have, for $t^{\prime} \in[0, M T), r_{L P}(t)=r_{L P}\left(t^{\prime}+T_{G}\right)=$ $\sum_{k=0}^{K-1} \sum_{l=0}^{L_{k}-1} \rho_{k, l} \tilde{s}_{k}^{(0)}\left(\left(t^{\prime}-\tau_{k}-\frac{l}{W}\right)_{M T}\right)+z_{L P}\left(t^{\prime}+T_{G}\right)$, where $(\cdot)_{M T} \equiv(\cdot) \cdot \bmod M T$. This novel approach, like the OFDM system, transmits the inverse Fourier transformed information symbols to the channels such that the ISI could be perfectly avoided with simple complexity.

B. Maximum A Posteriori Probability (MAP) Demodulation

Sampling the received low-pass equivalent signal $r_{L P}(t)$ at rate $W=2 / T_{c} \equiv 1 / T_{s}$ according to the Nyquist criterion, 
we have, for $k=0,1, \cdots, K-1, n=0,1, \cdots, N_{c}-1$, $m=0,1, \cdots, M-1$, and $q=0,1, r_{k}(n, m, q) \equiv r_{L P}\left(T_{G}+\right.$ $\left.\left(n T_{M}+m T_{c}+q T_{s}+\tau_{k}\right)_{M T}\right)=\sum_{k^{\prime}=0}^{K-1} \sum_{l=0}^{L_{k^{\prime}}-1} \rho_{k^{\prime}, l} p_{k^{\prime}}((n-$ $\left.\left.\alpha_{k, k^{\prime}, l, m, q}\right)_{N_{c}}\right) \ddot{b}_{k^{\prime}}^{(0)}\left(\left(m-\beta_{k, k^{\prime}, l, q}\right)_{M}\right)+z_{k}(n, m, q)$, where $z_{k}(n, m, q) \equiv z_{L P}\left(T_{G}+\left(n T_{M}+m T_{c}+q T_{s}+\tau_{k}\right)_{M T}\right)$ are independent and identically distributed (i.i.d.) complex-valued Gaussian random variables with zero mean; $(\cdot)_{I} \equiv(\cdot) \bmod I$ for any positive integer $I ; \alpha_{k, k^{\prime}, l, m, q}=\alpha \in \mathcal{Z}$ if $-\alpha T_{M} \leq$ $m T_{c}+q T_{s}+\tau_{k}-\tau_{k^{\prime}}-l T_{s}<-(\alpha-1) T_{M} ;$ and $\beta_{k, k^{\prime}, l, q}=\beta \in$ $\mathcal{Z}$ if $-\beta T_{c} \leq\left[q T_{s}-l T_{s}+\tau_{k}-\tau_{k^{\prime}}\right]_{T_{M}}^{+}<-(\beta-1) T_{c}$, where $[x]_{T_{M}}^{+} \equiv x-T_{M}\left\lfloor\frac{x}{T_{M}}\right\rfloor$, and $\lfloor x\rfloor$ returns the nearest integer of $x$ toward zero for any real number $x$. Taking $N_{c}$-point discrete Fourier transform (DFT) over $r_{k}(n, m, q)$ on $n$ gives

$$
\begin{aligned}
& R(k, m, q) \equiv \sum_{n=0}^{N_{\mathrm{c}}-1} r_{k}(n, m, q) e^{-j \frac{2 \pi}{N_{c}} n k} \\
= & \sum_{l=0}^{L_{k}-1} \rho_{k, l} N_{c} e^{-j \frac{2 \pi}{N_{c}} k \check{\alpha}_{l, m, q}} \ddot{b}_{k}^{(0)}\left(\left(m-\check{\beta}_{l, q}\right)_{M}\right) \\
+ & Z_{k}(k, m, q),
\end{aligned}
$$

where $Z_{k}(i, m, q)$ and $P_{k}(i)$ are the $N_{c}$-point DFT of $z_{k}(n, m, q)$ and $p_{k}(n)$ respectively over $n ; \check{\alpha}_{l, m, q} \equiv \alpha_{0,0, l, m, q}$; and $\check{\beta}_{l, q} \equiv \beta_{0,0, l, q}$. (1) is the consequence of employing the frequency-domain orthogonal signature sequence $p_{k}(n)=$ $e^{j \frac{2 \pi}{N_{c}} k n}$ whose $N_{c}$-point DFT is $P_{k}(i)=\left\{\begin{array}{ll}N_{c} & \text { if } k=i \\ 0 & \text { otherwise }\end{array}\right.$.

In (1), it can be seen that $R(k, m, q)$ is interfered by ISI introduced by frequency-selective channels instead of the MAI. However, this ISI can be avoided by taking $M_{\text {info-point }}$ DFT on (1) over $m$ provided $\check{\alpha}_{l, m, q}$ is irrelevant to $m$ for $m \in\left[M_{g}, M-1\right], l=0,1, \cdots, L_{k}-1$, and $q=0,1$.

Proposition 1: $\forall m \in\left[M_{g}, M-1\right]$, we have $\dot{\alpha}_{l, m, q}=0$ and $M_{g} \geq \check{\beta}_{l, q} \geq 0, \forall l=0,1, \cdots, L_{k}-1$, and $q=0,1$.

Proof: Because $m \in\left[M_{g}, M-1\right]$ and by the definition of $\check{\alpha}_{l, m, q}, 0 \leq M_{g} T_{c}+q T_{s}-l T_{s} \leq m T_{c}+q T_{s}-l T_{s} \leq$ $(M-1) T_{c}+q T_{s}-l T_{s}<M T_{c}=T_{M}$, as $q \in\{0,1\}, l \in$ $\left\{0,1, \cdots, L_{k}-1\right\}$, and $M_{g} \geq \max _{k=0,1, \cdots, K-1} L_{k} / 2$. Thus $\check{\alpha}_{l, m, q}=0$. Similar calculation gives $0 \leq \check{\beta}_{l, q} \leq M_{g}$. Q.E.D. By taking $M_{\text {info-point }}$ DFT over $R(k, m, q)$ for $m \in$ $\left[M_{g}, M-1\right]$, we have

$$
\begin{aligned}
\check{R}(k, i, q) & \equiv \sum_{m=M_{g}}^{M-1} R(k, m, q) e^{-j \frac{2 \pi}{M_{\text {info }}} m i} \\
& =\tilde{B}_{k}^{(0)}(i) H_{k}(i, q)+\check{Z}(k, i, q),
\end{aligned}
$$

where $\check{Z}(k, i, q) \equiv \sum_{m=M_{g}}^{M-1} Z_{k}(k, m, q) e^{-j \frac{2 \pi}{M M_{\text {info }}} m i}$ are complex-valued i.i.d. Gaussian random variables with zero mean; and $H_{k}(i, q) \equiv N_{c} \sum_{l=0}^{L_{k}-1} \rho_{k, l} e^{-j \frac{2 \pi}{M_{i n f o}} i \breve{\beta}_{l, q}}$. The optimal decoding according to the maximum $a$ posteriori probability (MAP) criterion is $\hat{B}_{k}^{(0)}(m)=$ $\arg \max _{b \in\{-1,1\}} \operatorname{Re}\left\{b \sum_{q=0}^{1} \check{R}(k, m, q) H_{k}^{*}(m, q)\right\}$, for $k=$ $0,1, \cdots, K-1$ and $m=0,1, \cdots, M_{\text {info }}-1$, where the superscript $*$ represents the complex-conjugate operation. Figure 3 (b) plots the optimal receiver of this approach.

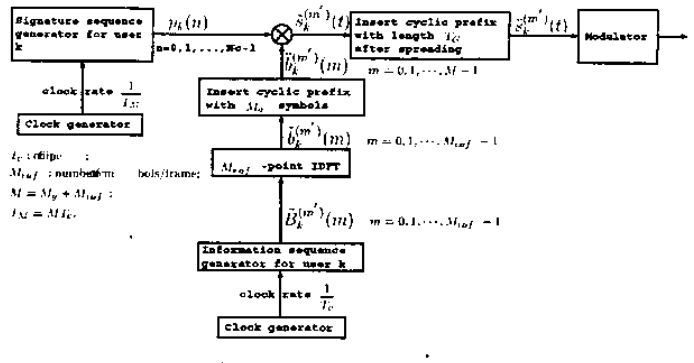

(a) Transmitter of user $k$.

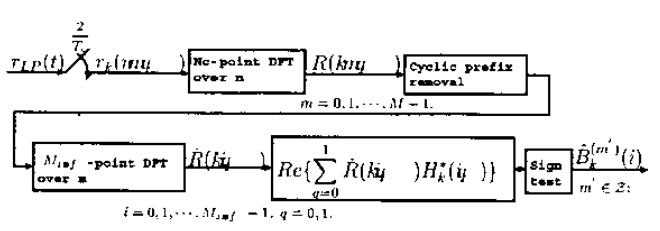

(b) Optimal receiver of user $k$.

Fig. 3. Transceiver of frequency-domain orthogonal multiuser communications systems with inverse transformation for user $k$.

\section{Numerical Analysis}

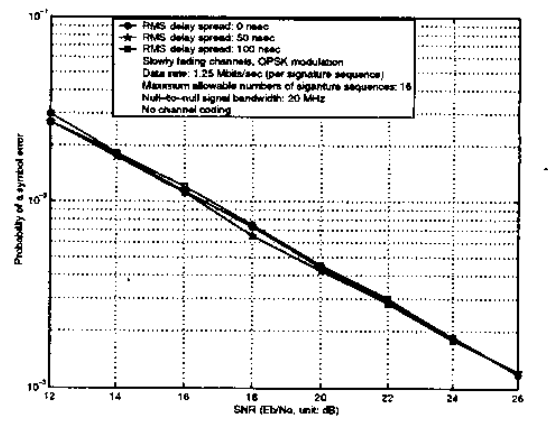

Fig. 4. Performance evaluation for FDOMC-IT systems.

We adopt the channel model extensively applied in wireless local area network (WLAN) [7] to justify our proposed system as we consider the slowly fading channels. In this model, the frequency-selective slowly, fading channel is modelled as the tapped delay line with tap spacing $T_{s}$, where $T_{s}$ is the sampling period. The number of taps is $\left\lceil 10 \frac{T_{R M S}}{T_{s}}\right\rceil$, where $T_{R M S}$ is the root-mean-square (RMS) value of the channel delay spread and $\lceil x\rceil$ is the operator to choose the smallest integer larger than $x$. In addition, the tap coefficients are i.i.d. complexvalued Gaussian random variables with exponentially decayed variance and zero mean.

We use the quadrature phase shift keying (QPSK) modula- 
tion as an example to verify the performance of our proposed system. $T_{c}$ is set to be $0.1 \mu \mathrm{sec}$ and $N_{c}=16$. Therefore, this system supports up to 16 users with symbol duration $T=N_{c} T_{\mathcal{c}}=1.6 \mu \mathrm{sec}$ and null-to-null bandwidth $W=20$ MHz. By setting $M_{g}=10$, the receiver is not interfered by ISI for channels with RMS delay spread smaller than 100 $n s e c$. As can be seen in Figure 4, wherein $E_{b}$ stands for the bit energy, the receiver's performance is not degraded by ISI introduced by frequency-selective channels.

Nevertheless, the possible frequency diversity inherited from wide-band systems is lost. By concentrating the energy of a symbol into some small portion of the frequency band, each symbol experiences the independent and identical flat fading channel when the coherent bandwidth of channel is not very small. Therefore, this kind of wide-band systems does not enjoy the frequency diversity inherited by wide-band systems.

A therapy to earn the frequency diversity back is to establish the correlations between symbols by employing channel coding and interleaving as IEEE 802.11a [8]. With the establishment of correlations between symbols, those deeply faded symbols may be possibly recovered by the other symbols experiencing independent and identical fading effects. Another approach to utilize the frequency diversity is to spread the symbol energy to the whole frequency band as the direct sequence spread spectrum (DSSS) communications systems. With this approach, each symbol could be recovered by the portion of the frequency band which does not deeply fade and therefore the frequency diversity is earned. In the sequel, we shall propose another frequency-domain orthogonal multiuser communications system with direct spreading to earn frequency diversity gain.

\section{FREQUenCy-Domain ORThogonal MUltiuser COMMUNICATIONS WITH DIRECT SPREADING \\ (FDOMC-DS) OVER FREQUENCY-SELECTIVE SLOWLY FADING CHANNELS}

\section{A. Signal Models and Assumptions}

The signal models and assumptions in FDOMC-DS are exactly the same as FDOMC-IT in Section II-A except that the information symbols for user $k$ in the $m^{\prime}$ frame are $\left\{\tilde{b}_{k}^{\left(m^{\prime}\right)}(m) \mid m=0,1, \cdots, M_{\text {info }}-1\right\}$ instead of $\left\{\tilde{B}_{k}^{\left(m^{\prime}\right)}(m) \mid m=0,1, \cdots, M_{\text {info }}-1\right\}$. That is, before the signal transmission, as shown in Figure 5(a), we do not perform IDFT on information symbols.

\section{B. Linear-Complexity Demodulation}

With the identical procedures in Section II-B, we obtain $\check{R}(k, m, q)=\tilde{B}_{k}^{(0)}(m) H_{k}(m, q)+\check{Z}(k, m, q)$, for $k=$ $0,1, \cdots, K-1, m=0,1, \cdots, M_{\text {info }}-1$, and $q=$ 0,1 as in (2). Defining three $M_{\text {info }} \times 1$ column vectors $\underline{\underline{R}}_{k, q}, \underline{\tilde{b}}_{k}$, and $\underline{\check{Z}}_{k, q}$ over $\mathcal{C}^{M_{\text {info }} \times 1}$ with the $n$th entries being $\check{R}(k, n, q), \tilde{b}_{k}^{(0)}(n)$, and $\check{Z}(k, n, q)$ respectively, we have $\underline{\underline{R}}_{k, q}=\mathbf{H}_{k, q} \mathbf{G}_{D F T} \underline{\underline{b}}_{k}+\underline{Z}_{k, q}$, where $\mathcal{C}$ is the field of complex numbers; $\mathbf{H}_{k, q}$ is the diagonal matrix with $H_{k}(n, q)$ being its entry in the $n$th row and the $n$th column; and $\mathbf{G}_{D F T}$ is the

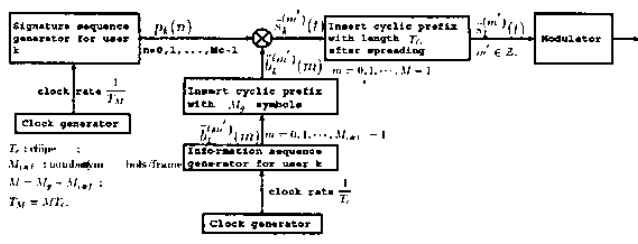

(a) Transmitter of user $k$.

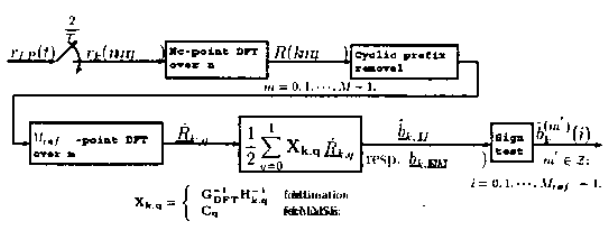

(b) Optimal receiver of user $k$.

Fig. 5. Transceiver of frequency-domain orthogonal multiuser communications systems with direct spreading for user $k$.

$M_{\text {info }} \times M_{\text {info }}$ square matrix whose entry in the $m$ th row and $n$th column is $e^{-j \frac{2 \pi}{M M_{i n f o}} m n}$.

Because $\check{Z}(k, m, q)$ are i.i.d. complex-valued zeromean Gaussian random variables, the maximum likelihood (ML) estimation of $\underline{\tilde{b}}_{k}$ is $\underline{\hat{b}}_{k, M L}=$ $\arg \max _{\underline{b} \in \mathcal{C}^{M_{\text {info }} \times 1}} \operatorname{Pr}\left(\underline{\check{R}}_{k, 0}, \underline{\check{R}}_{k, 1} \mid \underline{\tilde{b}}_{k}=\underline{b}\right)=$ $\frac{1}{2} \sum_{q=0}^{1} \mathbf{G}_{D F T}^{-1} \mathbf{H}_{k, q}^{-1} \underline{\underline{R}}_{k, q}=\underline{\tilde{b}}_{k}+\frac{1}{2} \sum_{q=0}^{1} \mathbf{G}_{D F T}^{-1} \mathbf{H}_{k, q}^{-1} \underline{\underline{Z}}_{k, q}$, which is the transmitted information $\tilde{b}_{k}$ contaminated by Gaussian noise. Therefore, this ML estimator is the "zero-forcing" equalizer to have zero ISI.

Due to the frequency selectivity of fading channels, the zero-forcing equalizer suffers the noise enhancement problem to degrade its performance. To solve this problem, we propose the linear minimum mean square error (LMMSE) estimation by designing the $M_{\text {info }} \times M_{\text {info }}$ square matrix $\mathbf{C}_{q} \in \mathcal{C}^{M_{\text {info }} \times M_{\text {info }}}$ whose entry in $m$ th row and $n$th column is $C_{q}(m-1, n-1) \in \mathcal{C}$ such that $\sum_{q=0}^{1} \sum_{m=0}^{M_{\text {info-1 }}-1} E \mid \sum_{n=0}^{M_{\text {info-1 }}-1} C_{q}(m, n) \check{R}(k, n, q)$ $\left.\tilde{b}_{k}^{(0)}(m)\right|^{2}$ is minimized. According to the orthogonality principle [9], we have

$$
C_{q}(m, n)=\frac{H_{k}^{*}(n, q) E\left|\tilde{b}_{k}^{(0)}(0)\right|^{2} e^{\frac{2 \pi}{M_{\text {info }}} m n}}{M_{\text {info }} E\left|\tilde{b}_{k}^{(0)}(0)\right|^{2}\left|H_{k}(n, q)\right|^{2}+E|\check{Z}(k, n, q)|^{2}} .
$$

These proposed ML and LMMSE estimators are simpler than the linear-complexity estimators proposed in [4] due to the introduction of $M_{g}$ guard symbols. In addition, as $\mathbf{G}_{D F T}^{-1} \underline{b}$

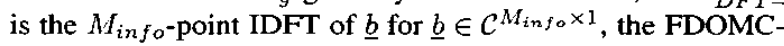
DS system with ML estimation has the same complexity as the FDOMC-IT one by shifting the $M_{\text {info-point IDFT from }}$ the receiver to the transmitter shown in Figure 5. 


\section{Numerical Analysis}

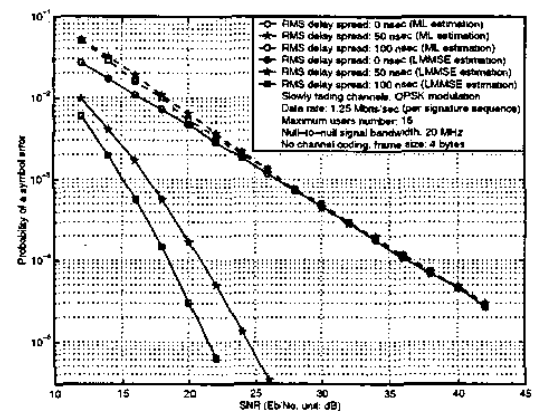

(a) Probability of symbol error.

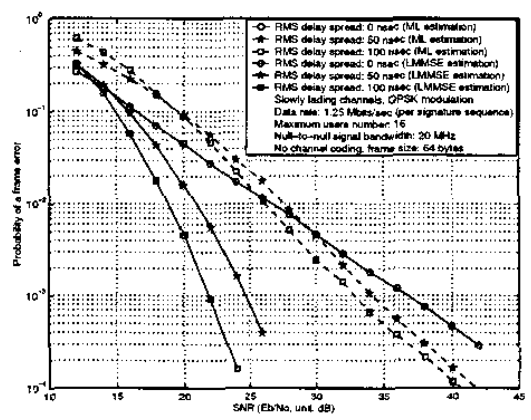

(b) Probability of frame error.

Fig. 6. Performance evaluation for FDOMC-DS systems over frequencyselective slowly fading channels.

We employ the identical simulation parameters as in Section II-C. As shown in Figure 6, both receiving structures earns frequency diversity to combat channel fading impairment and therefore are superior to the FDOMC-IT system. In addition, the diversity gain introduced by frequency-selective fading channels is much more efficiently utilized for LMMSE equalization than the zero-forcing estimation. This is the consequence of avoiding the noise enhancement suffered by the ML estimation.

\section{CONCLUSIONS}

We proposed two frequency-domain orthogonal multuser communications (FDOMC) systems: FDOMC-IT and FDOMC-DS over frequency-selective slowly fading channels which are not interfered by MAI even in the asynchronous channels. In addition, the FDOMC-IT system is further not interfered by ISI introduced by frequency-selective channels and can be optimally demodulated with low complexity. This is the consequence of concentrating the energies of independent information symbols into different sub-bands such that each sub-band experiences frequency-nonselective fading.
This approach does not earn the frequency diversity gain inherited from frequency-selective channels without efficiently establishing the correlation between information symbols.

By spreading the energies of independent information symbols into the whole frequency band, the FDOMC-DS system utilizes the frequency diversity to combat channel fading impairment such that it outperforms the FDOMC-IT one with similar complexity. Moreover, it was verified by the numerical analysis that the LMMSE estimation is much more efficient than the ML estimation to utilize the frequency diversity by taking the noise enhancement effects into consideration.

These two systems represent different transmission policies over frequency-selective slowly fading channels. When the channel impulse response (CIR) is available to the transmitter, the optimal power allocation follows the water-pouring policy [10] to maximize the channel capacity. In this case, the FDOMC-IT system which concentrates the symbol energies to some frequency sub-bands is easier to adopt its power allocation, signal constellations, and modulation types over different sub-bands according to the CIR to approach capacity. When the CIR is unknown to the transmitter, the FDOMC-DS system earns the frequency diversity in its best possibilities.

Both systems efficiently mitigate the ISI and MAI with complexity similar to the conventional approaches and are flexible to accommodate variable rates services. Each user can either change the modulation schemes, signal constellations, and so on or employ multiple signature sequences to fulfill high rate services without affecting the performance of the other users'. These valuable properties of the frequency-domain orthogonal multiuser communications systems suggest the applications to practical, multimedia, and advanced wireless communications systems.

\section{REFERENCES}

[1] Cha'o-Ming Chang and Kwang-Cheng Chen, "Frequency domain approach to multiuser detection in DS-CDMA communications," IEEE Communications Letters, vol. 4, no. 11, pp. 331-333, November 2000.

[2] S. Verdu, "Minimum probability of error for asynchronous Gaussian multiple-access channels," IEEE Trans. Inform. Theory, vol. IT-32, pp85-96, Jan. 1986.

[3] Bas W.'t. Hart, Richard D. J. Van Nee. Ramjee Prasad, "Performance degradation due to code tracking errors in spread-spectrum code-division multiple-access systems," IEEE Joumal on Selected Areas in Communications, vol. 14, No. 8, Oct. 1996.

[4] Cha'o-Ming Chang and Kwang-Cheng Chen, "Frequency-domain approach to DS-CDMA multiuser detection over frequency-selective slowly fading channels," in Proc. IEEE PIMRC. Sept. 2002, pp. 12801284

[5] John G. Proakis, Digital Communications, 2nd ed., New York: McGrawHill, 1989.

[6] Cha'o-Ming Chang, Mao Zeng, Kwang-Cheng Chen, and Vijay K. Bhargava, "Pilot-signal aided multiuser timing estimation in DS-CDMA communications," Proc. IEEE GLOBECOM, pp. 532-536, Dec. 1999.

[7] Bob $\mathrm{O}^{*} \mathrm{Hara}$ and Al Petrick, "The IEEE 802.11 handbook: A designer's companion," Standard Information Network IEEE Press, 1999.

[8] Supplement to IEEE Standard for Information technology - Part 11: Wireless LAN Medium Access Control (MAC) and Physical Layer (PHY) specifications: Higher-Speed Physical Layer in the $5 \mathrm{GHz}$ Band.

[9] H. Vincent Poor, An introduction to signal detection and estimation. Spring-Verlag, 1994

[10] S. Kasturia, J. T. Aslanis, and J. M. Cioffi, "Vector coding for partial response channels," IEEE Trans. Inform. Theory, vol. 36, pp. 741-762, July 1990 . 